\title{
Construction of a Multidimensional Potential Energy Surface from an Energy Spectrum
}

Robert Leonard Waterland

William \& Mary

John B. Delos

William \& Mary, jbdelos@wm.edu

Follow this and additional works at: https://scholarworks.wm.edu/aspubs

Part of the Physics Commons

\section{Recommended Citation}

Waterland, Robert Leonard and Delos, John B., Construction of a Multidimensional Potential Energy Surface from an Energy Spectrum (1984). Journal of Chemical Physics, 80(5), 2034-2041. https://doi.org/10.1063/1.446967

This Article is brought to you for free and open access by the Arts and Sciences at W\&M ScholarWorks. It has been accepted for inclusion in Arts \& Sciences Articles by an authorized administrator of W\&M ScholarWorks. For more information, please contact scholarworks@wm.edu. 


\title{
Construction of a multidimensional potential energy surface from an energy spectrum
}

\author{
R. L. Waterland and J. B. Delos \\ Department of Physics, The College of William and Mary, Williamsburg, Virginia 23185
}

(Received 8 July 1983; accepted 18 October 1983)

\begin{abstract}
A method for the systematic construction of a multidimensional potential energy surface from an energy spectrum is presented. We show that if the Hamiltonian and the energy spectrum satisfy certain conditions then there is an inversion technique which gives a potential energy function from the spectrum. The method proceeds via a sequence of canonical transformations and leads to a potential energy which is symmetric in all its variables. We test the procedure for one- and twodimensional examples and show that it gives reasonable results. However, the method does not give a unique result and we discuss two types of nonuniqueness exhibited by the procedure.
\end{abstract}

Given a Hamiltonian in quantum mechanics, there are many ways to calculate the spectrum of allowed discrete energy levels. We consider in this paper the inverse problem: Given a spectrum of energy levels, can we reconstruct the Hamiltonian? A general answer to this question is not presently available, but partial answers can be obtained if the problem is suitably restricted.

In particular, one-dimensional systems for which the semiclassical approximation is valid have been studied extensively and are now well understood. In the early years of quantum mechanics, shortly after the WKB method for calculating energy eigenvalues was developed, corresponding semiclassical inversion techniques were found for calculating a potential energy curve from the observed spectrum. ${ }^{\prime}$ These techniques are simple and reliable and they have had great success. Also for one-dimensional systems, there is a large literaure on fully quantum mechanical inversion techniques, which do not rely upon the semiclassical approximation. $^{2}$

However, very little is known about inversion procedures in more than one dimension. There is a substantial spectroscopic literature based on trial and error, ${ }^{3}$ and a classic mathematical paper by $\mathrm{Kac}^{4}$ ("Can One Hear the Shape of a Drum?"), but we know of only two other papers that deal with the systematic construction of an $N$-dimensional potential energy surface from a spectrum.

Gerber, Roth, and Ratner ${ }^{5}$ proposed an inversion method based upon a semiclassical self-consistent-field approximation. The SCF method involves the best separable approximation to the wave function and the semiclassical approximation then gives formulas for the energy levels that are sufficiently similar to one-dimensional formulas that an approximate type of RKR inversion can be used.

Clary $^{6 a}$ developed an iterative scheme for finding the potential energy based upon Percival and Pomphrey's meth$\mathrm{od}^{7}$ for finding vibrational eigenvalues. Simplifying somewhat, the essence of his idea is the following. Expressing the potential energy as a power series containing $M$ terms, one first has to guess the values of the coefficients in this series. Then invariant tori with quantized actions (eigentrajectories) are constructed. Each torus implies a relationship between the set of momenta $p$ and the set of coordinates $q$, and

$$
T[p(q)]+V(q)=E,
$$

where $E$ is the energy of the corresponding eigentrajectory. ${ }^{6 \mathrm{~b}}$ Now for each torus, let us select a particular point $q_{0}$ and let us denote $\widehat{E}$ as the observed energy for the corresponding quantum state. Then the expression

$$
V\left(q_{0}\right)=\widehat{E}-T\left[p\left(q_{0}\right)\right]
$$

can be used with $M$ distinct energies and their corresponding tori to determine $M$ coefficients in $V$. A new set of quantized tori is constructed, the new relationship $p(q)$ is again used in the above equation to obtain a new set of coefficents in $V$, and one iterates until convergence is (or is not) achieved.

In this paper, we develop a new approach to the inversion problem, using techniques involved in the Birkhoff Normal Form. We show that under certain conditions, the systematic procedures used to construct a spectrum from a Hamiltonian by the Birkhoff method can be done backwards, to obtain a Hamiltonian from a spectrum. We test this inversion method by carrying it out for one- and twodimensional systems, and we show that it gives reasonable results for the cases studied.

\section{REVIEW OF ONE-DIMENSIONAL INVERSION METHODS}

Our results will be clearer if we first briefly review the well-known one-dimensional semiclassical inversion techniques as used in molecular physics. In the Rydberg-KleinRees (RKR) method, a potential energy curve for a diatomic molecule is constructed pointwise from an energy spectrum. If vibrational and rotational energy levels are both known, then the RKR method is unambiguous; however, if the rotational levels are unknown and only the vibrational levels for a fixed rotational state are known, then only the differences between turning points can be recovered. In that case the potential energy function could be determined uniquely only if it were known to be symmetric. ${ }^{8}$

A very clear derivation is given by Landau and Lifshitz, ${ }^{9}$ and we state their result in a slightly different way. Given $m>0$ and given a finite function $\tau(E)>0$ on an interval $0 \leqslant E \leqslant \widehat{E}$, we define (for $V<\widehat{E}$ ) 


$$
q(V)=\frac{1}{2 \pi \sqrt{2 m}} \int_{0}^{V} \frac{\tau(E)}{(V-E)^{1 / 2}} d E
$$

Obviously $q(V) \geqslant 0$. One can show that $q(V)$ is a monotonically increasing function of $V$ if $\tau(E)$ is continuous and satisfies the integral inequality

$$
\tau(E) \geqslant \frac{1}{2(\sqrt{2}-1)} \lim _{\epsilon \rightarrow 0}\left[\sqrt{\epsilon} \int_{0}^{E-\epsilon} \frac{\tau(x) d x}{(E-x)^{3 / 2}}\right] .
$$

If $q(V)$ is a monotonic function of $V$, then the inverse function $V(q)$ exists for $q>0$, and we define for $q \leqslant 0$,

$$
V(q)=V(-q) \text {. }
$$

Then (theorem)

$$
\tau(E)=\sqrt{2 m} \int_{-q(E)}^{q(E)}[E-V(q)]^{-1 / 2} d q .
$$

This theorem has the following meaning: If we have a particle of known mass $m$ moving periodically in one dimension, and its period $\tau$ is known as a function of a parameter $E$ associated with its motion [and if $\tau(E)$ satisfies Eq. (2)], then there exists a Hamiltonian of the usual form,

$$
h(p, q)=\frac{p^{2}}{2 m}+V(q)
$$

with $V(q)$ a unique symmetric function, such that the period of motion under this Hamiltonian as a function of energy is precisely $\tau(E)$.

In quantum mechanics, the period of motion has no meaning, but in semiclassical mechanics, the period is interpolated from the discrete energy spectrum as

$$
\tau=\frac{d I}{d h} \approx \frac{2 \pi \hbar}{\Delta E}
$$

$I$ being an action variable and $\Delta E$ being the energy gap between adjacent levels.

Our results in $N$ dimensions are somewhat similar to the above. We also need an interpolation of the energy spectrum, and we also obtain a symmetric potential energy by a systematic process. On the other hand, the actual method of constructing the potential energy is quite different, and the result, unfortunately, is not unique.

\section{INVERSION OF THE BIRKHOFF PROCEDURE}

Earlier work ${ }^{10}$ has shown that a procedure invented by Birkhoff $^{\text {II }}$ (for incommensurable systems) and modified by Gustavson $^{12}$ (for commensurable systems) is a very good way to calculate an energy spectrum from a Hamiltonian. If the Hamiltonian is given in the form (we call it the "standard form")

$$
h=\sum_{i=1}^{N} \omega_{i}\left(p_{i}^{2}+q_{i}^{2}\right)+V\left(q_{1} \ldots q_{N}\right),
$$

where the frequencies are incommensurable, and $V\left(q_{1} \ldots q_{N}\right)$ is a power series containing cubic terms and higher, then Birkhoff showed that there exists a sequence of canonical transformations to new variables $\left\{P_{i}, Q_{i}\right\}$ such that the new Hamiltonian $H$ is a polynomial depending upon $P$ 's and $Q$ 's only in the combination $\omega_{i} J_{i}$, with

$$
\begin{array}{ll} 
& J_{i}=P_{i}^{2}+Q_{i}^{2}, \\
\text { i.e., } & \\
& H=H\left(\omega_{1} J_{1}, \ldots, \omega_{N} J_{N}\right) .
\end{array}
$$

Equation (6) is called the Birkhoff Normal Form. This Normal Form directly gives the energy spectrum by quantization in the new variables

$$
\begin{aligned}
& J_{i}=P_{i}^{2}+Q_{i}^{2} \leftrightarrow n_{i}+\frac{1}{2}, \\
& E_{n_{1} \ldots n_{N}}=H\left[\omega_{1}\left(n_{1}+\frac{1}{2}\right), \ldots, \omega_{N}\left(n_{N}+\frac{1}{2}\right)\right] .
\end{aligned}
$$

Our fundamental result is that the "dual" of this process can be carried out: from an interpolation of the energy spectrum in the form (8), there is a sequence of transformations that leads to a Hamiltonian in the "standard form" (4) with a symmetric potential energy.

More precisely, suppose we are given a set of energy levels of an $N$-dimensional system and that these levels form a regular pattern characterized by $N$ quantum numbers $n_{1} \ldots n_{N} \cdot{ }^{13}$ Suppose the pattern is such that the energy levels are given to good approximation by a polynomial expression in the quantum numbers [Eq. (8)] and suppose that the frequencies $\omega_{i}$ are incommensurable. We may then interpret the function $H$ as a Birkhoff Normal Form (6) which was quantized by the prescription (7). Then (theorem): there exists, and one can construct, a formal sequence of real canonical transformations which convert the polynomial $H$ into the standard form (4), in which the potential energy $V$ is a symmetric function of each of its variables

$$
V\left(q_{1} \cdots q_{i} \cdots q_{N}\right)=V\left(q_{1} \cdots-q_{i} \cdots q_{N}\right), \quad i=1, \ldots, N .
$$

The sequence begins by transforming terms of lowest degree and proceeds upwards through terms of higher degree. (There also exist other sequences of canonical transformations that construct unsymmetric potential energy functions, but coefficients of unsymmetric terms are not determined by this procedure.)

These results are proven below by methods similar to these used by Birkhoff.

(1) Given:

$$
\begin{aligned}
& H=\sum_{\text {even } l} H^{(l)}(\omega J), \\
& J_{i}=P_{i}^{2}+Q_{i}^{2}, \\
& H^{(0)}=0, \\
& H^{(2)}=\sum_{i} \omega_{i} J_{i},
\end{aligned}
$$

where $H^{(l)}$ is a homogeneous polynomial of degree $l$ in $P$ 's and $Q$ 's, and $\omega_{i}$ are incommensurable. ${ }^{14}$ Obviously terms of degree 2 and lower are already in standard form. We show that if all terms of degree less than $s$ are in standard form then the terms of degree $s$ can be put in standard form.

To prove this, define ${ }^{14}$

$$
F(P, q)=\sum_{i=1}^{N} P_{i} q_{i}+W^{(s)}(P, q),
$$

where $W^{(s)}$ is a homogeneous polynomial of degree $s$ in $P$ and $q$. The transformation equations generated by $F$ are 


$$
\begin{aligned}
& Q=q+\frac{\partial W^{(s)}}{\partial P}, \\
& p=P+\frac{\partial W^{(s)}}{\partial q}, \\
& h(p, q)=H(P, Q) .
\end{aligned}
$$

Substitution of Eqs. (11a), and (11b) into Eq. (11c) gives

$$
h\left(P+\frac{\partial W^{(s)}}{\partial q}, q\right)=H\left(P, q+\frac{\partial W^{(s)}}{\partial P}\right) .
$$

If Taylor expansions of $H$ and $h$ about $P$ and $q$ are performed and terms of equal degree in these expansions equated, the following set of equations is obtained:

$$
\begin{aligned}
& h^{(i)}(P, q)=H^{(i)}(P, q), \quad i<s, \\
& D W^{(s)}(P, q)=H^{(s)}(P, q)-h^{(s)}(P, q), i=s, \\
& h^{(i)}(P, q)=H^{(i)}(P, q) \quad i>s \\
& +\sum_{j, i} \frac{1}{j !}\left[\frac{\partial^{|j|} H^{(l)}}{\partial q^{(i)}}\left(\frac{\partial W^{(s)}}{\partial P}\right)^{j}-\frac{\partial^{|j|} h^{(l)}}{\partial P^{|j|}}\left(\frac{\partial W^{(s)}}{\partial q}\right)^{j}\right], \\
& j !=\prod_{i=1}^{N} j_{i} !, \quad|j|=\sum_{i=1}^{N} j_{i}, \\
& l-|j|+|j|(s-1)=i, \quad 1 \leqslant|j| \leqslant l<i, \\
& \quad l \geqslant 2, s \geqslant 3,
\end{aligned}
$$

where

$$
D=\sum_{i=1}^{N} \omega_{i}\left(P_{i} \frac{\partial}{\partial q_{i}}-q_{i} \frac{\partial}{\partial P_{i}}\right) .
$$

Equation (13a) shows that the order $s$ transformation leaves lower orders unaffected; Eq. (13b) must be solved for $W^{(s)}(P, q)$, and when this is done, Eq. (13c) gives the higher degree terms in the new Hamiltonian.

The solution to Eq. (13b) is

$$
W^{(s)}=D^{-1}\left(H^{(s)}-h^{(s)}\right) \text {. }
$$

It is most easily constructed by transforming to variables $\eta_{\mathrm{j}} \xi_{\mathrm{j}}$ such that

$$
\begin{aligned}
& P_{\mathrm{j}}=\frac{1}{\sqrt{2}}\left(\eta_{\mathrm{j}}+i \xi_{\mathrm{j}}\right), \\
& q_{\mathrm{j}}=\frac{i}{\sqrt{2}}\left(\eta_{\mathrm{j}}-i \xi_{\mathrm{j}}\right) .
\end{aligned}
$$

In these variables $D$ has the form

$$
D \rightarrow \widetilde{D}=i \sum_{j} \omega_{\mathrm{j}}\left(\xi_{\mathrm{j}} \frac{\partial}{\partial \xi_{\mathrm{j}}}-\eta_{\mathrm{j}} \frac{\partial}{\partial \eta_{\mathrm{j}}}\right)
$$

and its eigenfunctions are

$$
\Phi_{l_{1} \ldots l_{N}, m_{1} \cdots m_{N}}=\eta_{1}^{l_{1}} \eta_{2}^{l_{2}} \ldots \eta_{N}^{l_{N}} \xi_{1}^{m_{1}} \xi_{2}^{m_{2}} \ldots \xi_{N}^{m_{N}}
$$

with eigenvalues

$$
i \sum_{j} \omega_{j}\left(m_{\mathrm{j}}-l_{\mathrm{j}}\right)
$$

We require that

$$
\begin{gathered}
h^{(s)}=\sum_{s>3} V_{a_{1} a_{2} \cdots a_{N}}^{(s)} q_{1}^{a_{1}} q_{2}^{a_{2}} \cdots q_{N}^{a_{N}}, \\
\left(\sum_{j=1}^{N} a_{j}=s\right)
\end{gathered}
$$

where $V_{a_{1} \cdots a_{N}}^{(s)}$ are coefficients which are to be determined. When $H^{(s)}$ and $h^{(s)}$ are expressed as functions of $(\eta, \xi)$ it is found that they contain both range-space and null-space terms. ${ }^{15}$ For a solution to Eq. (14) to exist, it is necessary and sufficient to make the null-space terms in $h$ cancel those in $H$. Write

$\widetilde{H}^{(s)}=\widetilde{N}_{H}^{(s)}+\widetilde{R}_{H}^{(s)}, \quad \widetilde{h}^{(s)}=\widetilde{N}_{h}^{(s)}+\widetilde{R}_{h}^{(s)}$,

where $N$ and $R$ denote "the set of null-space terms" and "the set of range-space terms," respectively; then

$$
\begin{aligned}
& \widetilde{N}_{h}^{(s)}=\widetilde{N}_{H}^{(s)}, \\
& \widetilde{W}^{(s)}=\widetilde{D}^{-1}\left[\widetilde{R}_{H}^{(s)}-\widetilde{R}_{h}^{(s)}\right] .
\end{aligned}
$$

Equation (21) gives

$$
\frac{(N+s-1) !}{N !(s-1) !}
$$

relations for the undetermined $\left\{V^{(s)}\right\}$ of Eq. (19) in terms of known coefficients in $H$ and the $\left\{V^{(k)}\right\}$ from previous iterations. Equation (22) gives $\widetilde{W}^{(s)}(\eta, \xi)$, which can be used to generate higher order terms in the new Hamiltonian from Eq. (13c). This defines the iterative procedure, and completes the proof of the assertion made above Eq. (10).

(2) If $s$ is odd, all coefficients $V_{a_{1} \cdots a_{N}}^{(s)}$ are undetermined. For odd $s, H$ and $h$ are odd in at least one of the $P_{k}, q_{k}$. Hence, when they are reexpressed in terms of $\eta_{k} \xi_{k}$ they must remain of odd degree. But the only null space terms are powers of $\eta_{k} \xi_{k}$ i.e., they are of even degree. Hence Eqs. (21) and (22) put no restriction on odd degree terms and coefficients $V^{(s)}$ with $s$ odd are undetermined by this procedure.

(3) If $s$ is even, those coefficients $V_{a_{1} \cdots a_{N}}^{|s\rangle}$ for which any of the $a$ 's are odd are undetermined. (Proof is the same as above).

(4) If $s$ is even, then those coefficients $V_{a_{1} \cdots a_{N}}^{(s)}$ for which all $a$ 's are even are determined from $H^{(s)}$. Since

$$
q_{k}^{m}=\left[\frac{i}{\sqrt{2}}\left(\eta_{k}-i \xi_{k}\right)\right]^{m}
$$

if $m$ is even, the binomial expansion of $q_{k}^{m}$ contains a term proportional to

$$
\left(\eta_{k} \xi_{k}\right)^{m / 2}
$$

and this is in the null-space. From Eq. (21), its coefficient must be equal to that of the corresponding null-space term in $H$.

Since $V_{a_{1} \cdots a_{N}}^{(s)}$ are undetermined if any of the $a_{k}$ are odd, we may take them to be zero. Then the remaining $V_{a_{1} \cdots a_{N}}^{(s)}$ are uniquely specified by the iterative procedure.

The convergence properties of this inversion method ${ }^{16}$ have not been established; it is useful, therefore, to examine results when the procedure is carried to various levels.

\section{ONE DIMENSIONAL SYSTEMS}

As discussed earlier, there already exist successful onedimensional inversion methods. We discuss this case to illus- 
trate the new technique and to show that it leads to sensible results.

\section{A. General Inversion in one dimension}

Form is

For all one dimensional problems the Birkhoff Normal

$$
\begin{aligned}
H(P, Q)= & \frac{1}{2} \omega\left(P^{2}+Q^{2}\right)+H^{(2)}\left[\frac{1}{2} \omega\left(P^{2}+Q^{2}\right)\right]^{2} \\
& +H^{(3)}\left[\frac{1}{2} \omega\left(P^{2}+Q^{2}\right)\right]^{3} \\
& +H^{(4)}\left[\frac{1}{2} \omega\left(P^{2}+Q^{2}\right)\right]^{4}+\cdots
\end{aligned}
$$

where $\omega$ is the frequency of the unperturbed oscillator and $H^{(s)}$ are numerical coefficients. They could be found, e.g., by fitting parameters to the energy spectrum

$$
\begin{aligned}
E_{n}= & \left(n+\frac{1}{2}\right) \hbar \omega+H^{(2)}\left(n+\frac{1}{2}\right)^{2} \hbar^{2} \omega^{2} \\
& +H^{(3)}\left(n+\frac{1}{2}\right)^{3} \hbar^{3} \omega^{3}+H^{(4)}\left(n+\frac{1}{2}\right)^{4} \hbar^{4} \omega^{4}
\end{aligned}
$$

The generated Hamiltonian is required to be

$$
\begin{gathered}
h(p, q)=\frac{1}{2} \omega\left(p^{2}+q^{2}\right)+V^{(3)} q^{3}+V^{(4)} q^{4}+V^{(5)} q^{5} \\
+V^{(6)} q^{6}+V^{(7)} q^{7}+V^{(8)} q^{8}+\ldots
\end{gathered}
$$

Equations (13) were implemented using MACSYMA, ${ }^{17}$ a LISP-based language that permits analytical calculations.

Null-space terms appeared for even order transformations. According to theorem (2), $V^{(3)}, V^{(5)}$, and $V^{(7)}$ are undetermined, and they were arbitrarily set to zero. Resulting null-space terms were:

(i) Fourth degree terms

$$
\begin{aligned}
& \widetilde{N}_{h}^{(4)}=-\frac{3}{2} V^{(4)} \eta^{2} \xi^{2}, \\
& \widetilde{N}_{H}^{(4)}=-\omega^{2} H^{(2)} \eta^{2} \xi^{2} .
\end{aligned}
$$

(ii) Sixth degree terms

$$
\begin{aligned}
& \widetilde{N}_{h}^{(6)}=-\frac{s}{2} V^{(6)} i \eta^{3} \xi^{3} \\
& \tilde{N}_{H}^{(6)}=\left(-\omega^{3} H^{(3)}-3 \omega V^{(4)} H^{(2)}+\frac{V^{(4)^{2}}}{4 \omega}\right) i \eta^{3} \xi^{3} .
\end{aligned}
$$

(iii) Eighth degree terms

$$
\widetilde{N}_{h}^{(8)}=\frac{35}{8} V^{(8)} \eta^{4} \xi^{4}
$$

$\widetilde{N}_{H}^{(8)}=\left(\omega^{4} H^{(4)}-\frac{245}{16} V^{(4)^{2}} H^{(2)}+\frac{165}{8 \omega} V^{(4)} V^{(6)}-\frac{15 V^{(4)}}{32 \omega^{2}}\right)$

$$
\times \eta^{4} \xi^{4} \text {. }
$$

From Eq. (21)

$$
\widetilde{N}_{h}^{(s)}=\tilde{N}_{H}^{(s)} .
$$

The resulting equations are easily solved, giving

$$
\begin{aligned}
V^{(4)} & =\frac{2 \omega^{2} H^{(2)}}{3} \\
V^{(6)} & =\frac{2 \omega^{3}}{5}\left(H^{(3)}+\frac{17 H^{(2)^{2}}}{9}\right) \\
V^{(8)} & =\frac{4 \omega^{4}}{35}\left(2 H^{(4)}+11 H^{(2)} H^{(3)}+\frac{124}{18} H^{(2)^{3}}\right) .
\end{aligned}
$$

Hence the required Hamiltonian is (to the eighth degree)

$$
\begin{aligned}
& h(p, q) \\
& =\frac{1}{2} \omega\left(p^{2}+q^{2}\right)+\frac{2 \omega^{2} H^{(2)}}{3} q^{4}+\frac{2 \omega^{3}}{5}\left(H^{(3)}+\frac{17 H^{(2)^{2}}}{9}\right) q^{6} \\
& \quad+\frac{4 \omega^{4}}{35}\left(2 H^{(4)}+11 H^{(2)} H^{(3)}+\frac{124 H^{(2)^{3}}}{18}\right) q^{8}
\end{aligned}
$$

\section{B. Comparison of results of inversion by two methods}

Let us compare the results obtained above with those obtained through the more standard inversion technique. Our method of making this comparison is the following: We start from a certain zero-order potential energy

$$
V_{0}(q)=\frac{q^{2}}{2}-\frac{q^{3}}{20}
$$

which does not happen to be symmetric. From $V_{0}(q)$ we can compute $\tau(E)$, and from $\tau(E)$ we can compute a symmetric potential energy $V_{L}(q)$ using the method given by Landau and Lifschitz $\left(\mathrm{L}^{2}\right)$, as described in Sec. I of this article. Also from $V_{0}(q)$ we can calculate a Birkhoff normal form, and then invert it to obtain another symmetric potential energy $V_{B}(q)$.

\section{1. $L^{2}$ method}

$$
\tau(E)=\oint_{a}^{b} \frac{d q}{p(q)}
$$

where $p(q)$ is the classical momentum at position $q$ and $a, b$ are the classical turning points of the motion.

A particle moving in the potential

$$
V_{0}(q)=\frac{q^{2}}{2}-\frac{q^{3}}{20}
$$

is bound provided that the particle energy $E$ is less than 200/ 27. For such a particle

$$
\tau(E)=\sqrt{2} \int_{a}^{b} \frac{d q}{\sqrt{\left(E-\frac{q^{2}}{2}+\frac{q^{3}}{20}\right)}}, \quad(m=1) .
$$

Let the roots of the cubic equation in the denominator of the integrand be $\alpha, \beta$, and $\gamma$ where $\alpha>\beta>\gamma$. Substitution of

$$
q=\gamma+(\beta-\gamma) \sin ^{2} \varphi
$$

reduces the integral to a complete elliptic integral of the first kind, i.e.,

$$
\tau(E)=\frac{2 \sqrt{2} \sqrt[4]{3}}{\left\{\cos (\chi(E)-\pi / 6\}^{1 / 3}\right.} \int_{0}^{\pi / 2} \frac{d \varphi}{\sqrt{1-k^{2} \sin ^{2} \varphi}}
$$

where

$$
\chi(E)=\frac{1}{3} \cos ^{-1}\left(1-\frac{27 E}{100}\right)
$$

and

$$
k^{2}=\frac{\sin \chi(E)}{\cos (\chi(E)-\pi / 6)}
$$

In the $\mathrm{L}^{2}$ method, $\tau(E)$ determines only the difference between the two turning points. That difference can be found by direct solution of the cubic equation in the denominator of Eq. (32) and the result is 


$$
q_{1}(E)-q_{2}(E)=\frac{20}{\sqrt{3}} \sin \chi(E)
$$

so therefore the $\mathrm{L}^{2}$ method gives

$$
q\left(V_{L}\right)=\frac{10}{\sqrt{3}} \sin \chi\left(V_{L}\right)
$$

or, by use of Eq. (36),

$$
V_{L}(q)=\frac{100}{27}\left\{1-\cos \left[3 \sin ^{-1} \frac{\sqrt{3} q}{10}\right]\right\} .
$$

\section{Inverse Birkhoff method}

To apply the Birkhoff inversion, we require the Birkhoff-Gustavson normal form for the given Hamiltonian, i.e.,

$$
h_{0}=\frac{1}{2}\left(p^{2}+q^{2}\right)-q^{3} / 20, \quad(\omega=1) .
$$

Using the method of Swimm and Delos ${ }^{10}$ one readily obtains $H=0.5\left(P^{2}+Q^{2}\right)-2.34375 \times 10^{-3}\left(P^{2}+Q^{2}\right)^{2}$

$$
\begin{aligned}
& -8.25195 \times 10^{-6}\left(P^{2}+Q^{2}\right)^{3}-3.70102 \\
& \times 10^{-8}\left(P^{2}+Q^{2}\right)^{4}
\end{aligned}
$$

(to eighth degree). Comparison with Eq. (22) gives

$$
\begin{aligned}
& H^{(2)}=-9.37500 \times 10^{-3}, \\
& H^{(3)}=-6.60156 \times 10^{-5}, \\
& H^{(4)}=-5.92163 \times 10^{-7},
\end{aligned}
$$

and, using Eq. (30),

$$
\begin{aligned}
h= & 0.5\left(p^{2}+q^{2}\right)-6.25000 \times 10^{-3} q^{4}+4.00000 \times 10^{-5} q^{6} \\
& -5.08259 \times 10^{-7} q^{8} \quad \text { (to eighth degree). }
\end{aligned}
$$

\section{Comparison of methods}

In Fig. 1 we plot the result of the Landau technique [Eq. (40)] and that for the inverse Birkhoff technique [Eq. (44)]. Agreement is excellent for $q \leqslant 3.5$; the maximum error in this range is $3.2 \%$. As $q$ increases, the inaccuracy of the inverse Birkhoff curve increases and for $4.5 \leqslant q \leqslant 5.0$ it is a poor approximation to the exact $\mathrm{L}^{2}$ result. However the inverse Birkhoff result is everywhere a much more accurate curve

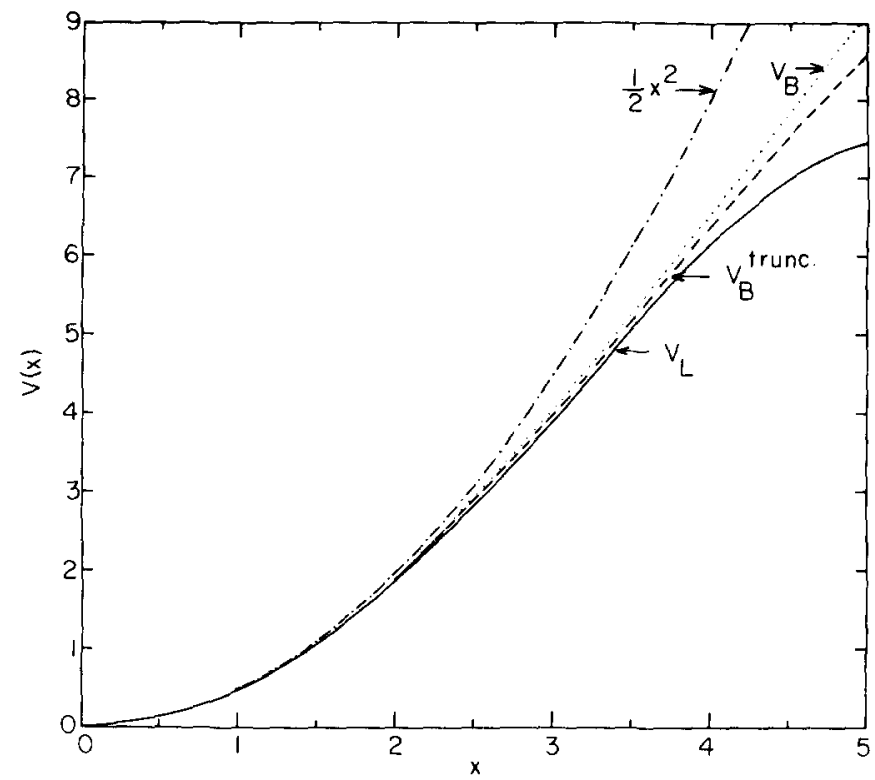

FIG. 1. Comparison of one dimensional inversion procedures: $V_{L}$ is the Landau result [Eq. (40)]; $V_{B}$ is the inverse Birkhoff result [Eq. (44)]; $V_{B}^{\text {trunc }}$ is the inverse Birkhoff result truncated to a quartic form.

than the pure harmonic oscillator curve which is plotted for reference.

(Somewhat surprisingly, the best fit is obtained by taking terms through $q^{4}$. Addition of the $q^{6}$ term reduces accuracy in the whole region $0<q \leqslant 5$; then addition of the $q^{8}$ term increases accuracy slightly. We have also plotted the inverse Birkhoff result truncated to a quartic form; for $q \leqslant 3.5$ the maximum error is $1.9 \%$ ).

\section{TWO DIMENSIONAL SYSTEMS}

In this section we apply the inverse Birkhoff method to the general two dimensional, incommensurable frequencies problem. To illustrate the technique we examine a particular example and demonstrate the consistency of the Birkhoff inversion method.

\section{A. General two dimensional incommensurable frequencies problem}

For all two dimensional incommensurable frequencies problems, we write the normal form using simpler notation:

$$
\begin{aligned}
& \boldsymbol{H}\left(\boldsymbol{P}_{1}, \boldsymbol{P}_{2}, \boldsymbol{Q}_{1}, Q_{2}\right) \\
& =\frac{1}{2} \omega_{1}\left(\boldsymbol{P}_{1}^{2}+Q_{1}^{2}\right)+\frac{1}{2} \omega_{2}\left(P_{2}^{2}+Q_{2}^{2}\right)+\beta_{1}\left[\frac{1}{2} \omega_{1}\left(P_{1}^{2}+Q_{1}^{2}\right)\right]^{2}+\beta_{2}\left[\frac{1}{2} \omega_{1}\left(P_{1}^{2}+Q_{1}^{2}\right) \frac{1}{2} \omega_{2}\left(P_{2}^{2}+Q_{2}^{2}\right)\right]+\beta_{3}\left[\frac{1}{2} \omega_{2}\left(P_{2}^{2}+Q_{2}^{2}\right)\right]^{2} \\
& \quad+\gamma_{1}\left[\frac{1}{2} \omega_{1}\left(P_{1}^{2}+Q_{1}^{2}\right)\right]^{3}+\gamma_{2}\left(\left[\frac{1}{2} \omega_{1}\left(P_{1}^{2}+Q_{1}^{2}\right)\right]_{\frac{1}{2}}^{2} \omega_{2}\left(P_{2}^{2}+Q_{2}^{2}\right)\right\}+\gamma_{3}\left\{\frac { 1 } { 2 } \omega _ { 1 } ( P _ { 1 } ^ { 2 } + Q _ { 1 } ^ { 2 } ) \left[\frac { 1 } { 2 } \omega _ { 2 } \left(P_{2}^{2}\right.\right.\right. \\
& \left.\left.\left.\quad+Q_{2}^{2}\right)\right]^{2}\right\}+\gamma_{4}\left[\frac{1}{2} \omega_{2}\left(P_{2}^{2}+Q_{2}^{2}\right)\right]^{3}+\cdots .
\end{aligned}
$$

The quantized energy spectrum would satisfy a corresponding formula, and the coefficients $\beta_{i}, \gamma_{i}, \ldots$ are assumed to be obtained from the spectrum.

The generated Hamiltonian is required to be 


$$
\begin{aligned}
h(p, q)= & \frac{1}{2} \omega_{1}\left(p_{1}^{2}+q_{1}^{2}\right)+\frac{1}{2} \omega_{2}\left(p_{2}^{2}+q_{2}^{2}\right)+V_{4,0}^{(4)} q_{1}^{4}+V_{3,1}^{(4)} q_{1}^{3} q_{2}+V_{2,2}^{(4)} q_{1}^{2} q_{2}^{2}+V_{1,3}^{(4)} q_{1} q_{2}^{3}+V_{0,4}^{(4)} q_{2}^{4} \\
& +V_{6,0}^{(6)} q_{1}^{6}+V_{5,1}^{(6)} q_{1}^{5} q_{2}+V_{4,2}^{(6)} q_{1}^{4} q_{2}^{2}+V_{3,3}^{(6)} q_{1}^{3} q_{2}^{3}+V_{2,4}^{(6)} q_{1}^{2} q_{2}^{4}+V_{1,5}^{(6)} q_{1} q_{2}^{5}+V_{0,6}^{(6)} q_{2}^{6} .
\end{aligned}
$$

Equations (13) were implemented using MACSYMA. Null-space terms appeared for even order transformations. Using theorem (2), $V_{3,1}^{(4)}, V_{1,3}^{(4)}, V_{5,1}^{(6)}, V_{3,3}^{(6)}$, and $V_{1,5}^{(6)}$ were set to zero. Resulting null-space terms were:

(i) Fourth degree terms

$$
\begin{aligned}
& \widetilde{N}_{h}^{(4)}=-\frac{3}{2} V_{4,0}^{(4)} \eta_{1}^{2} \xi_{1}^{2}+V_{2,2}^{(4)} \eta_{1} \xi_{1} \eta_{2} \xi_{2}-\frac{3}{2} V_{0,4}^{(4)} \eta_{2}^{2} \xi_{2}^{2}, \\
& \widetilde{N}_{H}^{(4)}=-\omega_{1}^{2} \beta_{1} \eta_{1}^{2} \xi_{1}^{2}+\omega_{1} \omega_{2} \beta_{2} \eta_{1} \xi_{1} \eta_{2} \xi_{2}-\omega_{2}^{2} \beta_{3} \eta_{2}^{2} \xi_{2}^{2} .
\end{aligned}
$$

(ii) Sixth degree terms

$$
\begin{aligned}
\widetilde{N}_{h}^{(6)}= & \left\{-3 \omega_{1} V_{4,0}^{(4)} \beta_{1}+\frac{V_{4,0}^{(4)}}{4 \omega_{1}}+\frac{\xi}{2} V_{6,0}^{(6)}\right\} i \eta_{1}^{3} \xi_{1}^{3}+\left\{-\frac{3}{2} \beta_{2} \omega_{2} V_{4,0}^{(4)}-\frac{\beta_{1} \omega_{1}^{2} \omega_{2}^{3} V_{2,2}^{(4)}}{\omega_{1} \omega_{2}\left(\omega_{2}-\omega_{1}\right)\left(\omega_{2}+\omega_{1}\right)}+\frac{\beta_{1} \omega_{1}^{4} \omega_{2} V_{2,2}^{(4)}}{\omega_{1} \omega_{2}\left(\omega_{2}-\omega_{1}\right)\left(\omega_{2}+\omega_{1}\right)}\right. \\
& -\frac{\beta_{2} \omega_{1}^{2} \omega_{2}^{3} V_{2,2}^{(4)}}{\omega_{1} \omega_{2}\left(\omega_{2}-\omega_{1}\right)\left(\omega_{2}+\omega_{1}\right)}+\frac{\beta_{2} \omega_{1}^{4} \omega_{2} V_{2,2}^{(4)}}{2 \omega_{1} \omega_{2}\left(\omega_{2}-\omega_{1}\right)\left(\omega_{2}+\omega_{1}\right)}+\frac{\omega_{1}^{4} \omega_{2}^{3} V_{2,2}^{(4)} 2}{4\left[\omega_{1} \omega_{2}\left(\omega_{2}-\omega_{1}\right)\left(\omega_{2}+\omega_{1}\right)\right]^{2}} \\
& \left.-\frac{\omega_{1}^{2} \omega_{2}^{5} V_{2,2}^{(4)} 2}{4\left[\omega_{1} \omega_{2}\left(\omega_{2}-\omega_{1}\right)\left(\omega_{2}+\omega_{1}\right)\right]^{2}}+\frac{3}{2} V_{4,2}^{(6)}\right\} \eta_{1}^{2} \xi_{1}^{2} \eta_{2} \xi_{2}+\left\{\frac{\beta_{2} \omega_{1}^{3} \omega_{2}^{2} V_{2,2}^{(4)}}{2 \omega_{1} \omega_{2}\left(\omega_{2}-\omega_{1}\right)\left(\omega_{2}+\omega_{1}\right)}\right. \\
& -\frac{\beta_{2} \omega_{1} \omega_{2}^{4} V_{2,2}^{(4)}}{2 \omega_{1} \omega_{2}\left(\omega_{2}-\omega_{1}\right)\left(\omega_{2}+\omega_{1}\right)}-\frac{\beta_{3} \omega_{1} \omega_{2}^{4} V_{2,2}^{(4)}}{\omega_{1} \omega_{2}\left(\omega_{2}-\omega_{1}\right)\left(\omega_{2}+\omega_{1}\right)}+\frac{\beta_{3} \omega_{1}^{3} \omega_{2}^{2} V_{2,2}^{(4)}}{\omega_{1} \omega_{2}\left(\omega_{2}-\omega_{1}\right)\left(\omega_{2}+\omega_{1}\right)} \\
& +\frac{\omega_{1}^{3} \omega_{2}^{4} V_{2,2}^{(4) 2} 2}{4\left[\omega_{1} \omega_{2}\left(\omega_{2}-\omega_{1}\right)\left(\omega_{2}+\omega_{1}\right)\right]^{2}} \\
& \left.-\frac{\omega_{1}^{5} \omega_{2}^{2} V_{2,2}^{(4) 2}}{4\left[\omega_{1} \omega_{2}\left(\omega_{2}-\omega_{1}\right)\left(\omega_{2}+\omega_{1}\right)\right]^{2}}-\frac{3}{2} \beta_{2} \omega_{1} V_{0,4}^{(4)}+\frac{3}{2} V_{2,4}^{(6)}\right\} \eta_{1} \xi_{1} \eta_{2}^{2} \xi_{2}^{2} \\
& +\left\{\frac{5}{2} V_{0,6}^{(6)}-3 \omega_{2} V_{0,4}^{(4)} \beta_{3}+\frac{V_{0,4}^{(4)}{ }^{2}}{4 \omega_{2}}\right\} i \eta_{2}^{3} \xi_{2}^{3},
\end{aligned}
$$

From Eq. (21)

$$
\widetilde{N}_{h}^{(s)}=\widetilde{N}_{H}^{(s)} .
$$

The resulting equations are easily solved giving:

$$
\begin{aligned}
& V_{4,0}^{(4)}=\frac{2 \beta_{1} \omega_{1}^{2}}{3}, \\
& V_{2,2}^{(4)}=\omega_{1} \omega_{2} \beta_{2}, \\
& V_{0,4}^{(4)}=\frac{2 \beta_{3} \omega_{2}^{2}}{3},
\end{aligned}
$$

$$
\begin{aligned}
& V_{6,0}^{(6)}=\frac{2 \omega_{1}^{3}}{5}\left\{\frac{17 \beta_{1}^{2}}{9}+\gamma_{1}\right\}, \\
& V_{4,2}^{(6)}=\frac{2 \omega_{1}^{2} \omega_{2}}{3}\left\{2 \beta_{1} \beta_{2}+\gamma_{2}+\frac{\left(3 \omega_{2}^{2}-2 \omega_{1}^{2}\right) \beta_{2}^{2}}{4\left(\omega_{2}^{2}-\omega_{1}^{2}\right)}\right\}, \\
& V_{2,4}^{(6)}=\frac{2 \omega_{1} \omega_{2}^{2}}{3}\left\{2 \beta_{2} \beta_{3}+\gamma_{3}+\frac{\left(2 \omega_{2}^{2}-3 \omega_{1}^{2}\right) \beta_{2}^{2}}{4\left(\omega_{2}^{2}-\omega_{1}^{2}\right)}\right\}, \\
& V_{0,6}^{(6)}=\frac{2 \omega_{2}^{3}}{5}\left\{\frac{17 \beta_{3}^{2}}{9}+\gamma_{4}\right\} .
\end{aligned}
$$

Hence the required Hamiltonian is (to eighth degree)

$$
\begin{aligned}
h(p, q)= & \frac{1}{2} \omega_{1}\left(p_{1}^{2}+q_{1}^{2}\right)+\frac{1}{2} \omega_{2}\left(p_{2}^{2}+q_{2}^{2}\right)+\frac{2 \beta_{1} \omega_{1}^{2}}{3} q_{1}^{4}+\omega_{1} \omega_{2} \beta_{2} q_{1}^{2} q_{2}^{2}+\frac{2 \beta_{3} \omega_{2}^{3}}{3} q_{2}^{4}+\frac{2 \omega_{1}^{3}}{5} \\
& \times\left\{\frac{17 \beta_{1}^{2}}{9}+\gamma_{1}\right\} q_{1}^{6}+\frac{2 \omega_{1}^{2} \omega_{2}}{3}\left\{2 \beta_{1} \beta_{2}+\gamma_{2}+\frac{\left(3 \omega_{2}^{2}-2 \omega_{1}^{2}\right)}{4\left(\omega_{2}^{2}-\omega_{1}^{2}\right)} \beta_{2}^{2}\right\} q_{1}^{4} q_{2}^{2}+\frac{2 \omega_{1} \omega_{2}^{2}}{3}\left\{2 \beta_{2} \beta_{3}+\gamma_{3}\right. \\
& \left.+\frac{\left(2 \omega_{2}^{2}-3 \omega_{1}^{2}\right)}{4\left(\omega_{2}^{2}-\omega_{1}^{2}\right)} \beta_{2}^{2}\right\} q_{1}^{2} q_{2}^{4}+\frac{2 \omega_{2}^{3}}{5}\left\{\frac{17 \beta_{3}^{2}}{9}+\gamma_{4}\right\} q_{2}^{6} .
\end{aligned}
$$




\section{B. An example: Birkhoff inversion for the Hénon-Helles Hamlitonian}

Swimm and Delos ${ }^{10}$ have calculated the Birkhoff normal form for the Hénon-Heiles ${ }^{18}$ Hamiltonian,

$h_{0}=\frac{1}{2} \omega_{1}\left(p_{1}^{2}+q_{1}^{2}\right)+\frac{1}{2} \omega_{2}\left(p_{2}^{2}+q_{2}^{2}\right)+\frac{\lambda}{\omega_{1} \omega_{2}^{1 / 2}} q_{1}^{2} q_{2}+\frac{\lambda \eta}{\omega_{2}^{3 / 2}} q_{2}^{3}$

with

$$
\omega_{1}=1.3, \quad \omega_{2}=0.7, \lambda=-0.1, \quad \eta=0.1 .
$$

They obtained

$$
\begin{aligned}
H= & \frac{1.3}{2}\left(P_{1}^{2}+Q_{1}^{2}\right)+\frac{0.7}{2}\left(P_{2}^{2}+Q_{2}^{2}\right)-1.56185 \times 10^{-3}\left[\frac{1}{2}\left(P_{2}^{2}+Q_{2}^{2}\right)\right]^{2}-1.023323 \times 10^{-2}\left[\frac{1}{4}\left(P_{1}^{2}+Q_{1}^{2}\right)\left(P_{2}^{2}+Q_{2}^{2}\right)\right] . \\
& -5.80199 \times 10^{-3}\left[\frac{1}{2}\left(P_{1}^{2}+Q_{1}^{2}\right)\right]^{2}-1.092 \times 10^{-5}\left[\frac{1}{2}\left(P_{2}^{2}+Q_{2}^{2}\right)\right]^{3}-1.7719 \times 10^{-4}\left[\frac{1}{8}\left(P_{2}^{2}+Q_{2}^{2}\right)^{2}\left(P_{1}^{2}+Q_{1}^{2}\right)\right] \\
& -2.7327 \times 10^{-4}\left[\frac{1}{8}\left(P_{2}^{2}+Q_{2}^{2}\right)\left(P_{1}^{2}+Q_{1}^{2}\right)^{2}\right]-8.625 \times 10^{-5}\left[\frac{1}{2}\left(P_{1}^{2}+Q_{1}^{2}\right)\right]^{3} \quad \text { (to eighth degree). }
\end{aligned}
$$

Comparison of Eq. (58) with Eq. (45) gives

$$
\begin{aligned}
& \beta_{1}=-3.43313 \times 10^{-3}, \\
& \beta_{2}=-1.12453 \times 10^{-2}, \\
& \beta_{3}=-3.18745 \times 10^{-3}, \\
& \gamma_{1}=-3.92581 \times 10^{-5}, \\
& \gamma_{2}=-2.30997 \times 10^{-4}, \\
& \gamma_{3}=-2.78163 \times 10^{-4}, \\
& \gamma_{4}=-3.18367 \times 10^{-5},
\end{aligned}
$$

and, using Eq. (56),

$$
\begin{aligned}
h= & \frac{1.3}{2}\left(p_{1}^{2}+q_{1}^{2}\right)+\frac{0.7}{2}\left(p_{2}^{2}+q_{2}^{2}\right)-3.86799 \times 10^{-3} q_{1}^{4}-1.02332 \times 10^{-2} q_{1}^{2} q_{2}^{2} \\
& -1.04123 \times 10^{-3} q_{2}^{4}-1.494 \times 10^{-5} q_{1}^{6}-8.160 \times 10^{-5} q_{1}^{4} q_{2}^{2}-4.192 \times 10^{-5} q_{1}^{2} q_{2}^{4}-1.74 \times 10^{-6} q_{2}^{6} .
\end{aligned}
$$

To within the accuracy of the Birkhoff and inverse Birkhoff procedures, this Hamiltonian (60) should give the same spectrum as does the Hénon-Heiles Hamiltonian ${ }^{19}$ (57).

\section{UNIQUENESS}

As noted earlier, the present method does not uniquely determine the potential energy surface. There are two distinct types of nonuniqueness in the inverse Birkhoff technique.

The inverse Birkhoff method does not determine asymmetric terms in the potential energy function. In this regard it is similar to other inversion methods. The one dimensional $\mathbf{R K R}^{1,20}$ method requires both rotational and vibrational data for uniqueness; if the rotational spectrum is unknown the RKR method can only give a symmetric potential energy curve. Likewise Clary ${ }^{6 a}$ notes that his inversion "does not appear to be appropriate for determining potentials containing odd powers of the coordinates," since it does not incorporate rotational data. However, Gerber et al. ${ }^{5}$ did include the rotational part of the spectrum, so they were able to obtain a unique result.

A second type of nonuniqueness arises from the observation that the generating function $\widetilde{W}$ is not uniquely deter- mined. Referring to Eq. (22) it is apparent that arbitrary nullspace terms could be added to $\widetilde{W}$. In all previous work using the BGNF, ${ }^{10,12}$ such terms have been omitted. By direct calculation we have found that addition of arbitrary null-space terms to the generating function results in changes in the symmetric part of the potential function. Such problems also exist in the calculation of a normal form, but nevertheless the work of Gustavson, of Swimm and Delos, and of Jaffé and Reinhardt indicates that the omission of the arbitrary nullspace terms leads to good results. Perhaps these extra terms are, in some sense, insignificant. At present, this type of nonuniqueness is not understood.

\section{CONCLUSION}

We have presented a method for constructing a multidimensional potential energy surface from an energy spectrum. In this method, the energy spectrum is fitted to a truncated Birkhoff Gustavson Normal Form, and then a sequence of canonical transformations is applied to the truncated normal form which transform it into a Hamiltonian of standard form (4). Symmetric potential energy terms are de- 
termined by this procedure; asymmetric terms are not, and they are set to zero.

The method has been applied to the general cases in one and two dimensions, and analytic formulas relating the coefficients of the normal form to those of the generated symmetric potential have been derived. We have applied the technique to two examples and it has been found to give reasonable results.

\section{ACKNOWLEDGMENTS}

We thank Don Noid for performing a numerical computation and Carl Andersen for his assistance during the writing of the MACSYMA programs. The contributions of the Mathlab Group at the Laboratory for Computer Science, MIT in making MACSYMA available are appreciated. This research was supported by the National Science Foundation.

'J. L. Dunham, Phys. Rev. 41, 721 (1932); I. Sandeman, Proc. R. Soc. Edinburgh 60, 210 (1940); W. R. Jarmain, Can. J. Phys. 38, 217 (1960); H. W. Wooley, J. Chem. Phys. 37, 1307 (1962); R. Rydberg, Z. Phys. 73, 376 (1931); O. Klein, ibid. 76, 226 (1932); A. L. G. Rees, Proc. R. Soc. London Ser. A 59, 998 (1947).

${ }^{2}$ C. E. Frobërg, Phys. Rev. 72, 519 (1947); E. A. Hylleraas, Phys. Rev. 74, 48 (1948); L. D. Faddeev, J. Math. Phys. 4, 72 (1963); V. Bargman, Phys. Rev. 75, 301 (1949); Rev. Mod. Phys. 21, 488 (1949); T. M. Gel'fand, and B. M. Levitan, Dokl. Akad. Nauk S.S.S.R. 77, $559(1951)$; R. Jost and W. Kohn, K. Dan. Vidensk Selsk, Mat.-Fys. Medd. 27 (1953); Phys. Rev. 87, 977 (1952); 88, 832 (1952); N. Levinson, Phys. Rev. 75, 1445 (1949). These results are compactly expressed in R. G. Newton, Scattering Theory of Waves and Particles (McGraw-Hill, New York, 1966), pp. 610-632.

${ }^{3}$ K. Machida and J. Overend, J. Chem. Phys. 50, 4429 (1969); 51, 2535 (1969); D. F. Smith and J. Overend, ibid. 53, 2411 (1970); 57, 523 (1972); R. G. Parr and J. E. Brown, ibid. 49, 4849 (1968); 54, 3429 (1971); A. C. Jeannotte, C. Marcott, and J. Overend, ibid. 68, 2076 (1978); K. Scanlon, I. Suzuki, and J. Overend, ibid. 74, 3735 (1981).
${ }^{4}$ M. Kac, Am. Math. Monthly 73, 1 (1966).

${ }^{5}$ R. B. Gerber, R. M. Roth, and M. A. Ratner, Mol. Phys. 44, 1335 (1981). ${ }^{6(2)}$ D. C. Clary, Chem. Phys. 41, 307 (1979)

${ }^{6|b|}$ The relationship between $q$ and $p$ is not a single-valued function, but $q$ and $p$ are each single-valued functions of angle variables $\theta$. Properly $p\left(q_{0}\right)$ really means $\left[p\left(\theta_{0}\right), q\left(\theta_{0}\right)\right]$, where $\theta_{0}$ is some chosen value of the angle variables.

${ }^{7}$ I. C. Percival and N. Pomphrey, J. Phys. B 9, 3131 (1976).

${ }^{8}$ For diatomic molecules, the potential energy is not symmetric, and it cannot be determined from the vibrational spectrum alone.

${ }^{9}$ L. D. Landau and E. M. Lifshitz, Mechanics (Pergamon, Oxford, 1976), pp. 27-29.

${ }^{10}$ R. T. Swimm and J. B. Delos, J. Chem. Phys. 71, $1706(1979) ;$ C. Jaffee and W. P. Reinhardt, ibid. 77, 5191 (1982); W. P. Reinhardt and D. Farrelly, J. Phys. Coll. Suppl. 11, 29 (1982).

${ }^{11} \mathrm{G}$. D. Birkhoff, Dynamical Systems (American Mathematics Society, New York, 1966), Vol. IX.

${ }^{12}$ F. G. Gustavson, Astron. J. 71, 670 (1966).

${ }^{13} \mathrm{~A}$ set of energy levels with this property is known as a regular spectrum; Hamiltonian systems can also display irregular spectra-see I. C. Percival, J. Phys B 6, L229 (1973); D. W. Noid, M. L. Koszykowski, and R. A. Marcus, Annu. Rev. Phys. Chem. 32, 267 (1981); M. V. Berry, J. Phys. A 10, 2083 (1977).

${ }^{14}$ In subsequent equations, $p, q, P, Q$ mean "the set of $p$," "the set of $q$," "the set of $P$," and "the set of $Q$," respectively, and $W J$ means "the set of products $W_{i} J_{i}, "$

${ }^{15} \alpha$ is in the null-space of operator $A$ if $A \alpha=0$, otherwise $\alpha$ is in the range space of $A$. For incommensurable frequencies, the only null-space terms of $\widetilde{D}$ are $\Phi_{\beta_{1} \beta_{2} \cdots \beta_{k}}=\left(\eta_{1} \xi_{1}\right)^{\beta_{1}}\left(\eta_{2} \xi_{2}\right)^{\beta_{2}} \cdots\left(\eta_{k} \xi_{k}\right)^{\beta_{k}}$ for all positive integers $k$.

${ }^{16} \mathrm{The}$ convergence properties of the Birkhoff Normal form have been established by C. L. Siegel, Ann. Math. 42, 806 (1941); Math. Ann. 128, 144 (1945); Vorlesung über Himmelsmechanik (Springer, Berlin, 1957). The Normal form converges if the coefficients in the Hamiltonian satisfy a certain infinite set of conditions; these conditions being rarely satisfied, the method diverges in general. For a discussion see J. Moser, Stable and Random Motions in Dynamical Systems, Ann. Math. Studies (Princeton University, Princeton, 1973) Vol. 77.

${ }^{17}$ R. Bogen, MACSYMA Reference Manual (The Mathlab Group, MIT, 1977).

${ }^{18} \mathrm{M}$. Hénon and C. Heiles, Astron. J. 69, 73 (1964).

${ }^{19} \mathrm{~A}$ test of this, though not a definite one, was made for us by D. Noid; neglecting the terms proportional to $q_{1}^{6}$ and to $q_{2}^{6}$, he computed the spectrum associated with Eq. (60). The resulting spectrum is in fact very close to that of Eq. (57).

${ }^{20}$ See for example E. A. Mason and L. Monchick, Adv. Chem. Phys. 12, 329 (1967). 